\title{
Metabolic flexibility and oxidative capacity independently associate with insulin sensitivity in individuals with newly diagnosed type 2 diabetes
}

\author{
Maria Apostolopoulou ${ }^{1,2}$ - Klaus Strassburger ${ }^{2,3}$ - Christian Herder ${ }^{1,2}$. \\ Birgit Knebel $^{2,4}$ • Jörg Kotzka ${ }^{2,4}$. Julia Szendroedi ${ }^{1,2,5}$ • Michael Roden ${ }^{1,2,5}$. \\ for the GDS group
}

Received: 29 April 2016 / Accepted: 8 June 2016 / Published online: 4 July 2016

(C) Springer-Verlag Berlin Heidelberg 2016

\begin{abstract}
Aims/hypothesis Both inherited and acquired insulin resistance have been associated with abnormal muscle mitochondrial function. At whole-body level, maximal oxygen uptake $\left(\dot{V} \mathrm{O}_{2 \max }\right)$ and/or metabolic flexibility (as given by $\Delta \mathrm{RQ}$ ) reflect certain features of mitochondrial function. This study tests the hypotheses (1) that $\dot{V} \mathrm{O}_{2 \max }$ and $\Delta \mathrm{RQ}$ correlate tightly with each other and with insulin sensitivity and (2) that glycaemia, lipidaemia or subclinical inflammation would explain such relationships.

Methods Near-normoglycaemic individuals with type 2 diabetes mellitus $(n=136)$ with a short known disease duration ( $<12$ months) underwent cycling spiroergometry, indirect calorimetry and hyperinsulinaemic-euglycaemic clamp tests.

Results Both $\dot{V} \mathrm{O}_{2 \max }(r=0.39, p<0.0001)$ and $\Delta \mathrm{RQ}$ $(r=0.32, p<0.0001)$ correlated positively with whole-body

The GDS group consists of M. Roden (speaker), A. E. Buyken, J. Eckel, G. Geerling, H. Al-Hasani, C. Herder, A. Icks, J. Kotzka, O. Kuss, E. Lammert, J. Lundbom, K. Müssig, P. Nowotny, W. Rathmann, J. Szendroedi, D. Ziegler and their co-workers, who are responsible for the design and performance of the GDS.
\end{abstract}

Electronic supplementary material The online version of this article (doi:10.1007/s00125-016-4038-9) contains peer-reviewed but unedited supplementary material, which is available to authorised users.

Michael Roden

michael.roden@ddz.uni-duesseldorf.de

1 Institute for Clinical Diabetology, German Diabetes Center, Leibniz Center for Diabetes Research at Heinrich-Heine University Düsseldorf, Düsseldorf, Germany

2 German Center for Diabetes Research (DZD), München-Neuherberg, Germany insulin sensitivity, even after adjusting for anthropometric variables, glycaemia and glucose-lowering medication, but not after adjusting for NEFA. $\dot{V} \mathrm{O}_{2 \max }$ further correlated negatively with circulating high-sensitivity $\mathrm{C}$-reactive protein concentration. However, $\dot{V} \mathrm{O}_{2 \max }$ did not relate to $\Delta \mathrm{RQ}$, even after adjusting for whole-body insulin sensitivity.

Conclusions/interpretation Oxidative capacity and metabolic flexibility are independent determinants of insulin sensitivity but are influenced by circulating NEFA in recent-onset type 2 diabetes.

ClinicalTrial.gov registration no: NCT01055093

Keywords Energy metabolism · Inflammation · Insulin sensitivity $\cdot$ Mitochondria $\cdot$ Muscle
3 Institute for Biometrics and Epidemiology, German Diabetes Center, Leibniz Center for Diabetes Research at Heinrich-Heine University Düsseldorf, Düsseldorf, Germany

4 Institute for Clinical Biochemistry and Pathobiochemistry, German Diabetes Center, Leibniz Center for Diabetes Research at Heinrich-Heine University Düsseldorf, Düsseldorf, Germany

5 Department of Endocrinology and Diabetology, Medical Faculty, Heinrich-Heine University Düsseldorf, c/o German Diabetes Center at Heinrich-Heine University, Auf dem Hennekamp 65, 40225 Düsseldorf, Germany 


$\begin{array}{ll}\text { Abbreviations } \\ \text { FFM } & \text { Fat-free mass } \\ \text { FLI } & \text { Fatty liver index } \\ \text { GDS } & \text { German Diabetes Study } \\ \text { hsCRP } & \text { High-sensitivity C-reactive protein } \\ \text { REE } & \text { Resting energy expenditure } \\ \text { SNP } & \text { Single-nucleotide polymorphism } \\ \dot{V} \mathrm{O}_{2 \max } & \text { Maximal oxygen consumption }\end{array}$

\section{Introduction}

Muscle insulin resistance results from inherited and acquired abnormalities. The latter comprise excess lipid availability with activation of the diacylglycerol-protein kinase $\mathrm{C}$ pathway [1] abnormalities in mitochondrial function [2] and glucose toxicity, at least in individuals with overt type 2 diabetes mellitus.

'Metabolic flexibility' defines the ability to switch from lipid to glucose oxidation during insulin stimulation as measured by an increase in the RQ $(\Delta \mathrm{RQ})[3]$. On the other hand, maximal oxygen uptake $\left(\dot{V} \mathrm{O}_{2 \max }\right)$ during exhaustive physical activity corresponds to muscle mitochondrial oxidative capacity, as reflected by ADP-stimulated oxidative phosphorylation at submaximal energy demand during exercise [2]. Whether the impaired metabolic flexibility in type 2 diabetes results from disturbed mitochondrial oxidation rather than from diminished muscle glucose uptake is as yet unclear. If impaired metabolic flexibility is the result of impaired mitochondrial oxidation, $\triangle \mathrm{RQ}$ should correlate with $\dot{V} \mathrm{O}_{2 \max }$, as demonstrated for muscle ATP synthase flux and phosphocreatine recovery [4]. A lower $\Delta R Q$ could also reflect impaired insulinmediated suppression of lipolysis in adipose tissue, leading to ectopic fat deposition and insulin resistance [5].

We examined a larger cohort of near-normoglycaemic participants with type 2 diabetes mellitus and a known disease duration of less than 12 months to test the hypothesis that $\dot{V} \mathrm{O}_{2 \max }$ and $\Delta$ RQ tightly correlate both with each other and with insulin sensitivity.

\section{Methods}

\section{Study design}

Individuals aged 18-69 years were recruited from the prospective German Diabetes Study (GDS) within the first year of their diagnosis of type 2 diabetes [6]. They consented to the trial protocol (ClinicalTrial.gov registration no. NCT01055093) which was approved by the ethics board of Heinrich-Heine University Düsseldorf. We included 136 participants for whom cycling spiroergometry, indirect calorimetry and clamp data were available.

\section{Spiroergometry}

Each participant performed an incremental exhaustive exercise test on a cycle ergometer (Ergoline Ergometrics 900 Bitz Germany) at 60 revolutions $/$ min $1 \mathrm{~h}$ after a standardised meal. Respiratory gas exchange was measured by open-air spirometry (MasterScreen CPX; Jäger/Viasys, Hoechberg, Germany).

\section{Indirect calorimetry}

Resting energy expenditure (REE) and RQ were calculated from $\mathrm{O}_{2}$ consumption and $\mathrm{CO}_{2}$ production using an automated respiratory gas analyser with a ventilated hood system and Vmax Encore 29n (CareFusion, Hoechberg, Germany). The RQ was measured as the quotient $\dot{V} \mathrm{CO}_{2} / \dot{V} \mathrm{O}_{2}$ at rest and during the last $30 \mathrm{~min}$ of the clamp. The $\Delta \mathrm{RQ}$ is given as the increase in RQ during the clamp [3].

\section{Hyperinsulinaemic-euglycaemic clamps}

All participants refrained from strenuous physical activity and withdrew their diabetes medication for 3 days, alcohol consumption for $24 \mathrm{~h}$ and food intake for $10-12 \mathrm{~h}$ prior to the modified Botnia clamp test combined with $\left[6,6-{ }^{2} \mathrm{H}\right]$ glucose infusion [6]. Whole-body insulin sensitivity was assessed as the $M$ value, given by the difference between mean glucose infusion rate during the last $30 \mathrm{~min}$ of the clamp and the glucose space correction, calculated as $\left(\mathrm{G}_{180}-\mathrm{G}_{150}\right) / 30$, with $\mathrm{G}_{180}$ and $\mathrm{G}_{150}$ representing the glucose levels at 180 and $150 \mathrm{~min}$, respectively.

Laboratory analyses All measurements were carried on the first study day in participants who had fasted for 10-12 h [6]. Blood glucose concentration was measured by the hexokinase method (EPOS 5060 analyser; Eppendorf, Hamburg, Germany), serum concentrations of insulin and C-peptide chemoluminimetrically, NEFA microfluorimetrically [1] $(n=78)$, triacylglycerols on a Cobas C311 analyser (Roche Diagnostics, Mannheim, Germany), and high-sensitivity C-reactive protein (hsCRP) on a Roche Hitachi 912 analyser (Roche Diagnostics, Mannheim, Germany). Explorative genotyping for single-nucleotide polymorphisms (SNPs) of genes known to affect mitochondrial function and/or insulin sensitivity (PPARG, NDUFB6, PGC1 $\alpha$ [also known as PPARGC1A], PPARA, ADRB2 and FTO) was performed in 134 participants (see electronic supplementary material [ESM]). Hepatic steatosis was assessed using the fatty liver index (FLI), a validated surrogate of liver fat content. 


\section{Statistical analyses}

Data are presented as means and SDs or medians (25th/75th percentiles) as appropriate. Variables with a skewed distribution ( $M$ value, insulin and triacylglycerols) were log-transformed before analysis. The logit transformation $\left[\operatorname{logit}(x)=\log _{e}(x / 1-x)\right]$ was applied to the FLI. Pearson correlation analyses, adjusted for sex, were used to study associations between $\dot{V} \mathrm{O}_{2 \max }, \Delta \mathrm{RQ}, M$ value, C-peptide concentration and anthropometric variables. Multiple regression analyses were carried out to adjust for potential confounders. Values of $p \leq 5 \%$ from two-sided tests were considered to indicate significant differences. Analyses were performed using SAS version 9.3 (SAS Institute, Cary, NC, USA).

\section{Results}

\section{Participants' characteristics}

The participants were overweight to slightly obese moderately insulin resistant and near-normoglycaemic. Women had higher BMI, fasting NEFA and hsCRP values, while men had a higher fat-free mass (FFM), REE and $\dot{V} \mathrm{O}_{2 \max }$ (Table 1). The allelic and genotyping frequencies of tested SNPs are provided in ESM Table 1.

\section{Associations of insulin sensitivity with $\dot{V} \mathbf{O}_{2 \max }$ and $\Delta \mathrm{RQ}$}

$\triangle$ RQ correlated with $M$ values across all participants (Fig. 1a) in men $(r=0.34, p=0.0007)$ and only marginally in women $(r=0.28, p=0.056) . \dot{V} \mathrm{O}_{2 \max }$ also correlated with $M$ values (Fig. 1b), fasting C-peptide concentration $(r=-0.35$, $p<0.001)$ and WHR $(r=-0.28, p=0.0007)$, but not with fasting lipid oxidation $(r=0.14, p=0.10) . \dot{V} \mathrm{O}_{2 \max }$ correlated with $M$ values in men $(r=0.43, p<0.0001)$, but correlated only marginally in women $(r=0.29, p=0.055) . \Delta$ RQ did not relate to $\dot{V} \mathrm{O}_{2 \max }$ either without (Fig. 1c) or with adjustment for $M$ values $(r=-0.07, p=0.41)$. $M$ values related to $\mathrm{C}$ peptide level $(r=-0.52, p<0.0001)$, WHR $(r=-0.42$, $p<0.0001)$ and FFM $(r=-0.46, p<0.0001)$. Neither $\Delta$ RQ, $\dot{V} \mathrm{O}_{2 \max }$ nor $M$ value correlated with $\mathrm{HbA}_{1 \mathrm{c}}$ concentration.

NEFA correlated positively with $\triangle \mathrm{RQ}$ but not with $\dot{V} \mathrm{O}_{2 \max }$ or $M$ value (ESM Fig. 1a-c). hsCRP correlated negatively with $\dot{V} \mathrm{O}_{2 \max }(r=-0.35, p=0.0004)$ and $M$ value $(r=-0.19, p=0.03)$ (ESM Fig. 1d, e).

Fasting RQ correlated negatively with NEFA $(r=-0.23$ $p=0.03)$, but not with $M$ value $(r=0.01, p=0.86)$ or $\dot{V} \mathrm{O}_{2 \max }$ $(r=-0.002, p=0.97)$.
FLI correlated negatively with $M$ value $(r=-0.59$ $p<0.0001), \dot{V} \mathrm{O}_{2 \max }(r=-0.42, p<0.0001)$ and $\Delta \mathrm{RQ}$ $(r=-0.21, p=0.01)$, but not with NEFA $(r=0.13, p=0.24)$.

Associations of tested SNPs with $\dot{V} \mathrm{O}_{2 \max } \Delta \mathrm{RQ}$ and $M$ value are provided in ESM Table 2. Only FTO (allelic variant TA/AA vs TT) correlated inversely with $\dot{V} \mathrm{O}_{2 \max }$.

\section{Multiple regression analysis for determinants of insulin sensitivity}

$\Delta \mathrm{RQ}$ and $\dot{V} \mathrm{O}_{2 \max }$ were associated with insulin sensitivity across the whole group when adjusted for sex $(\triangle \mathrm{RQ}$ : $\left.\beta=1.92 p=0.0001 \dot{V} \mathrm{O}_{2 \max }: \beta=0.04 p<0.0001\right)$. Additional adjustments for age, anthropometric variables, hsCRP, FLI, medication or combinations of these did not affect the associations of $\triangle$ RQ and $\dot{V} \mathrm{O}_{2 \max }$ with $M$ values (ESM Table 3 ). However, further adjusting for NEFA abolished the correlations of $\triangle \mathrm{RQ}$ and $\dot{V} \mathrm{O}_{2 \max }$ with $M$ values in the subcohort of 78 participants for whom NEFA concentrations were available (ESM Table 3, Models 8 and 9).

\section{Discussion}

This study found independent associations of $\Delta \mathrm{RQ}$ and $\dot{V} \mathrm{O}_{2 \max }$ with insulin sensitivity in metabolically well-controlled individuals with recent-onset type 2 diabetes. However $\triangle \mathrm{RQ}$ and $\dot{V} \mathrm{O}_{2 \text { max }}$ did not correlate with each other, suggesting that they may reflect distinct metabolic features.

The correlation between $\triangle \mathrm{RQ}$ and insulin sensitivity extends previous findings in longstanding type 2 diabetes to individuals with a short known disease duration [4]. Furthermore $\Delta R Q$ related positively, whereas fasting RQ related negatively, to serum NEFA, reflecting higher fasting lipid oxidation. Lipid elevation can give rise to diacylglycerols, which contribute to insulin resistance [1]. However, NEFA concentrations vary substantially in obese and T2D patients and may be higher during short-term fasting even in insulin-sensitive humans [7]. Nevertheless, NEFA elevation during lipid infusion or prolonged fasting leads to insulin resistance with a subsequent reduction in muscle oxidative capacity [8]. Prolonged fasting identifies abnormal muscle mitochondrial function as a consequence rather than a cause of human insulin resistance [9]. Finally, metabolic flexibility might also reflect insulin-mediated suppression of NEFA and thereby the insulin sensitivity of adipose tissue [5]. $\triangle \mathrm{RQ}$ also correlated negatively with FLI but not with hsCRP.

In overweight non-obese individuals the association of $\dot{V} \mathrm{O}_{2 \text { max }}$ with insulin sensitivity has been explained mainly by intrahepatic lipid content [10]. The present study found a similar association in people with recently diagnosed type 2 diabetes but detected no link with hepatic steatosis. According to 
Table 1 Characteristics of male and female participants with newly diagnosed type 2 diabetes

\begin{tabular}{|c|c|c|c|}
\hline & Men & Women & $p$ values \\
\hline$n$ & 93 & 43 & \\
\hline Age (years) $(n=136)$ & $51.4 \pm 10.5$ & $51.9 \pm 9.9$ & 0.939 \\
\hline $\operatorname{BMI}\left(\mathrm{kg} / \mathrm{m}^{2}\right)(n=136)$ & $30.9 \pm 6.1$ & $33.4 \pm 7.5$ & $0.046^{*}$ \\
\hline WHR $(n=136)$ & $0.99 \pm 0.06$ & $0.90 \pm 0.05$ & $<0.0001^{*}$ \\
\hline Fasting blood glucose $(\mathrm{mmol} / \mathrm{l})(n=133)$ & $7.21 \pm 2.00$ & $7.27 \pm 1.33$ & 0.217 \\
\hline Fasting serum insulin $(\mathrm{pmol} / \mathrm{l})(n=135)$ & $96.54(60.42,152.79)$ & $113.9(80.56,162.51)$ & 0.175 \\
\hline $\mathrm{HbA}_{1 \mathrm{c}}(\%)(n=136)$ & $6.5 \pm 0.9$ & $6.4 \pm 1.0$ & 0.861 \\
\hline $\mathrm{HbA}_{1 \mathrm{c}}(\mathrm{mmol} / \mathrm{mol})(n=136)$ & $47.0 \pm 10.3$ & $46.8 \pm 11.2$ & 0.861 \\
\hline$M$ value $\left(\mathrm{mg}[\mathrm{kg} \mathrm{BW}]^{-1} \min ^{-1}\right)(n=136)$ & $6.7(4.9,8.4)$ & $6.1(4.2,7.5)$ & 0.118 \\
\hline$M$ value $\left(\mathrm{mg}[\mathrm{kg} \mathrm{FFM}]^{-1} \min ^{-1}\right)(n=136)$ & $9.4(7.6,11.9)$ & $10.8(7.4,13.0)$ & 0.261 \\
\hline RQ during fasting $(n=136)$ & $0.80 \pm 0.06$ & $0.81 \pm 0.05$ & 0.491 \\
\hline RQ during clamp $(n=136)$ & $0.92 \pm 0.06$ & $0.92 \pm 0.06$ & 0.496 \\
\hline$\Delta \mathrm{RQ}(n=136)$ & $0.11 \pm 0.06$ & $0.12 \pm 0.06$ & 0.959 \\
\hline$\dot{V} \mathrm{O}_{2 \max } / \mathrm{kg} \mathrm{BW}\left(\mathrm{ml} \mathrm{min}_{-1}[\mathrm{~kg} \mathrm{BW}]_{-1}\right)(n=136)$ & $21 \pm 4$ & $16 \pm 3$ & $<0.0001^{*}$ \\
\hline$\dot{V} \mathrm{O}_{2 \max } / \mathrm{kg} \mathrm{FFM}\left(\mathrm{ml} \mathrm{min}{ }_{-1}[\mathrm{~kg} \mathrm{FFM}]_{-1}\right)(n=136)$ & $30 \pm 5$ & $28 \pm 5$ & 0.058 \\
\hline REE during fasting (kJ/day) $(n=136)$ & $8259 \pm 1363$ & $6882 \pm 1154$ & $<0.0001^{*}$ \\
\hline REE during clamp (kJ/day) $(n=136)$ & $8660 \pm 1204$ & $7213 \pm 1075$ & $<0.0001^{*}$ \\
\hline FFM $(\mathrm{kg})(n=136)$ & $68 \pm 10$ & $51 \pm 6$ & $<0.0001^{*}$ \\
\hline $\mathrm{FM}(\mathrm{kg})(n=136)$ & $32 \pm 12$ & $39 \pm 13$ & $0.001 *$ \\
\hline Fasting serum NEFA $(\mathrm{mmol} / \mathrm{l})(n=78)$ & $0.61 \pm 0.18(n=50)$ & $0.81 \pm 0.33(n=28)$ & $0.003 *$ \\
\hline Fasting serum LDL-cholesterol $(\mathrm{mmol} / \mathrm{l})(n=135)$ & $3.52 \pm 0.85$ & $3.68 \pm 1.04$ & 0.301 \\
\hline Fasting serum triacylglycerol $(\mathrm{mmol} / \mathrm{l})(n=136)$ & $1.44(1.15,2.32)$ & $1.50(1.07,2.07)$ & 0.263 \\
\hline Fasting lipid oxidation $\left(\mathrm{mg}[\mathrm{kg} \mathrm{BW}]^{-1} \min ^{-1}\right)(n=136)$ & $0.80 \pm 0.28$ & $0.71 \pm 0.24$ & 0.08 \\
\hline $\operatorname{hsCRP}(\mathrm{nmol} / \mathrm{l})(n=132)$ & $23.81(11.43,43.81)$ & $32.38(13.33,71.43)$ & 0.023 \\
\hline
\end{tabular}

Data are mean \pm SD. $M$ values, insulin levels and blood triacylglycerol concentrations are presented as medians (25th, 75 th percentiles) ${ }^{*} p<0.05$

BW, body weight; FM, fat mass

recent hypotheses, diminished muscle mitochondrial function and/or adipose tissue inflammation could lead to increased metabolite flux to the liver and decreased lipid oxidation, and thereby promote steatosis and insulin resistance [5]. This study found no association of $\dot{V} \mathrm{O}_{2 \max }$ with fasting lipid oxidation, but an inverse correlation with hsCRP, suggesting that a role of inflammatory pathways in insulin sensitivity is already present in individuals with newly diagnosed type 2 diabetes.
Although hyperglycaemia may contribute to the development of insulin resistance and oxidative stress interfering with mitochondrial function this study failed to detect any association of insulin sensitivity and variables of mitochondrial function with glucometabolic control. This is likely to be due to the good metabolic control and short duration of diabetes.

Of the tested genes known to affect energy metabolism, only the presence of the A allele in the rs9939609 SNP in
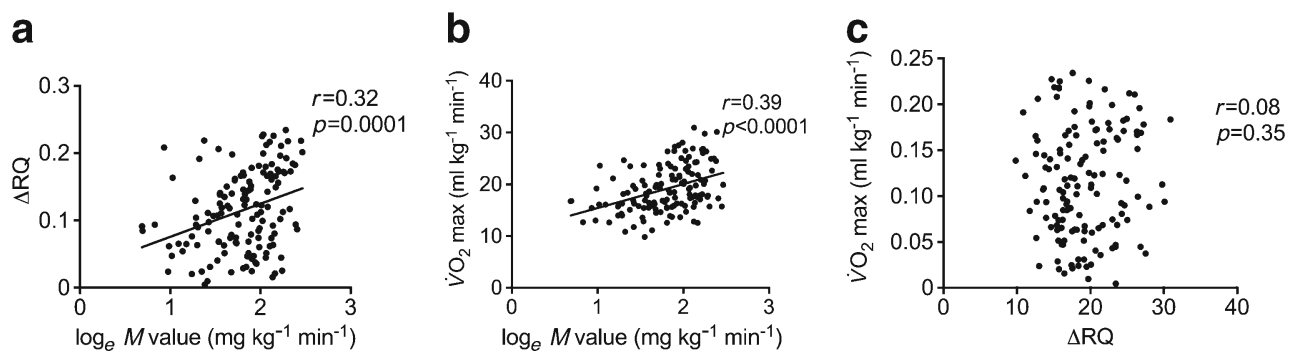

Fig. 1 Association between $\Delta \mathrm{RQ}($ a) $) \dot{V} \mathrm{O}_{2 \text { max }}$ related to whole-body weight (b) and the $\log _{e}$ of the $M$ value related to whole-body weight in participants with type 2 diabetes. (c) Relationship between $\dot{V} \mathrm{O}_{2 \max }$ related to whole-body weight and $\triangle \mathrm{RQ}$ among participants with type 2 diabetes 
the FTO gene was associated with a lower $\dot{V} \mathrm{O}_{2 \max }$, although this disappeared after Bonferroni correction. This gene has been shown to link obesity with an increased risk of type 2 diabetes [11]. Although the sample size may be too small to draw valid conclusions, these data do not support a central role of genetic regulation of the interaction between energy metabolism and insulin sensitivity.

Of note, $\dot{V} \mathrm{O}_{2 \max }$ and $\Delta \mathrm{RQ}$ correlated with insulin sensitivity even after adjusting for various variables in our multiple regression analysis models. Thus, impaired $\dot{V} \mathrm{O}_{2 \max }$ and $\Delta \mathrm{RQ}$ are likely to be early abnormalities in the course of diabetes contributing to the progression of insulin resistance and hepatic steatosis. It should be noted that adjusting for circulating NEFA abolished the association of $\dot{V} \mathrm{O}_{2 \max }$ and $\triangle \mathrm{RQ}$ with insulin sensitivity, underlining the superior importance of lipidaemia in insulin resistance. Although the only-marginal associations of $\dot{V} \mathrm{O}_{2 \max }$ and $\Delta \mathrm{RQ}$ with $M$ value in women may suggest a sex-specific difference, NEFA elevation has comparable effects on insulin resistance and muscle energy metabolism in both men and women [1]. Thus, the observed differences in the associations could also be due to the lower statistical power of the analyses of the female study population $(n=43)$.

The strengths of this study are the simultaneous analyses of clinical measures of mitochondrial function combined with the gold standard method for assessing insulin sensitivity in well-phenotyped individuals with type 2 diabetes. This study however, also has limitations. First, $\dot{V} \mathrm{O}_{2 \max }$ depends not only on mitochondrial function, but also on oxygen supply as determined by cardiorespiratory fitness. Second, $\triangle \mathrm{RQ}$ is influenced by fasting glycaemia and specifically by NEFA concentrations, which exhibit individual variation during the clamp procedure. In an attempt to overcome these limitations, the associations of $\dot{V} \mathrm{O}_{2 \max }$ and $\Delta \mathrm{RQ}$ with insulin sensitivity were also examined after multiple adjustments.

\section{Conclusions}

In near-normoglycaemic individuals with recently diagnosed type 2 diabetes $\dot{V} \mathrm{O}_{2 \max }$ and $\Delta \mathrm{RQ}$ independently associate with insulin sensitivity. As only adjustment for circulating NEFA abolished these associations, lipotoxic mechanisms could contribute to the impairment of metabolic plasticity and maximal oxidative capacity that occurs early in the course of type 2 diabetes.

Funding This work was supported by the Ministry of Science and Research of the State of North Rhine-Westphalia (MIWF NRW) and the German Federal Ministry of Health (BMG). The study was supported in part by a grant from the Federal Ministry for Research (BMBF) to the German Center for Diabetes Research (DZD e.V.), and by grants from the Helmholtz Alliance Imaging and Curing Environmental Metabolic Diseases (ICEMED), German Research Foundation (DFG, SFB1116, project B05) and Schmutzler-Stiftung. The funding sources had no input into the design and conduct of this study, the collection, analysis and interpretation of the data, or the preparation, review or approval of the paper.

Duality of interest The authors declare that there is no duality of interest associated with this manuscript.

Contribution statement MA wrote the manuscript and researched and collected the data. KS performed the statistical analysis and revised the manuscript. BK and JK performed the SNP and metabolomic analysis and revised the manuscript. $\mathrm{CH}$ researched data and revised the manuscript. JS and MR designed the study, researched data and revised the manuscript. All authors have read and approved the final version of this manuscript. MR is the guarantor of this work and, as such, had full access to all the data in the study and takes responsibility for the integrity of the data and the accuracy of the data analysis.

\section{References}

1. Szendroedi J, Yoshimura T, Phielix E et al (2014) Role of diacylglycerol activation of $\mathrm{PKC} \theta$ in lipid-induced muscle insulin resistance in humans. Proc Nat Acad Sci 111:9597-9602

2. Szendroedi J, Phielix E, Roden M (2012) The role of mitochondria in insulin resistance and type 2 diabetes mellitus. Nat Rev Endocrinol 8:92-103

3. Kelley DE, Mandarino LJ (2000) Fuel selection in human skeletal muscle in insulin resistance: a reexamination. Diabetes 49:677-683

4. van de Weijer T, Sparks LM, Phielix E et al (2013) Relationships between mitochondrial function and metabolic flexibility in type 2 diabetes mellitus. PloS One 8:e51648

5. Shulman GI (2014) Ectopic fat in insulin resistance, dyslipidemia, and cardiometabolic disease. New Engl J Med 371:1131-1141

6. Szendroedi J, Saxena A, Weber KS et al (2016) Cohort profile: the German Diabetes Study (GDS). Cardiovasc Diabetol 15:59

7. Karpe F, Dickmann JR, Frayn KN (2011) Fatty acids, obesity, and insulin resistance: time for a reevaluation. Diabetes 60:2441-2449

8. Brehm A, Krssak M, Schmid AI, Nowotny P, Waldhausl W, Roden M (2006) Increased lipid availability impairs insulin-stimulated ATP synthesis in human skeletal muscle. Diabetes 55:136-140

9. Hoeks J, van Herpen NA, Mensink M et al (2010) Prolonged fasting identifies skeletal muscle mitochondrial dysfunction as consequence rather than cause of human insulin resistance. Diabetes 59:2117-2125

10. Haufe S, Engeli S, Budziarek P et al (2010) Cardiorespiratory fitness and insulin sensitivity in overweight or obese subjects may be linked through intrahepatic lipid content. Diabetes 59:1640-1647

11. Frayling TM, Timpson NJ, Weedon MN et al (2007) A common variant in the FTO gene is associated with body mass index and predisposes to childhood and adult obesity. Science 316:889-894 